\title{
Raynaud Phenomenon of the Nipple Successfully Treated With Nifedipine and Gabapentin
}

\author{
Humberto Gallego, MD; Joseph S. Aleshaki, DO
}

\section{PRACTICE POINTS}

- Raynaud phenomenon of the nipple may be accompanied by lancinating pain of the breast in addition to nipple pain reminiscent of postherpetic neuralgia.

- Associated breast pain is particularly distressing for breastfeeding women, particularly primiparous mothers with children intolerant to formula.

- In women with Raynaud phenomenon accompanied by lancinating breast pain, consider a trial of pregabalin.

To the Editor:

Raynaud phenomenon is characterized by vasospasm of arterioles causing intermittent ischemia of the digits. The characteristic triphasic color change presents first as a dramatic change in skin color from normal to white, as the vasoconstriction causes pallor secondary to ischemia. This change is followed by a blue appearance, as cyanosis results from the deoxygenated venous blood. Finally, reflex vasodilation and reperfusion manifest as a red color from erythema. Several cases have been reported describing Raynaud phenomenon affecting the nipples of breastfeeding women. ${ }^{1-5}$ This vasospasm results in episodic nipple pain manifesting from breastfeeding and exposure to cold. If it is not appropriately treated, the pain's severity causes affected women to stop breastfeeding. We report a case of vasospasm of the nipple in which the patient experienced nipple pain and a separate lancinating pain that radiated through the breasts.

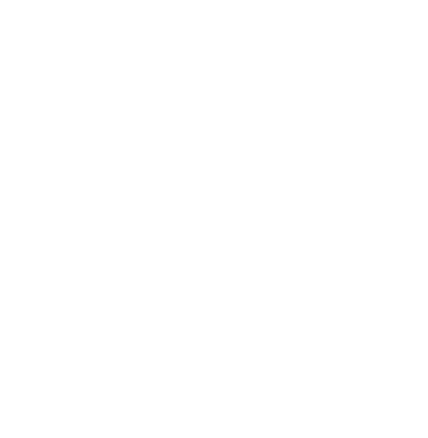

A 36-year-old woman presented with excruciating nipple and breast pain 3 weeks after delivering her first child. She had no history of smoking or Raynaud phenomenon. The nipple pain was triggered upon breastfeeding and exposure to cold. During these episodes, the nipples would initially blanch white, then turn purple and finally a deep red. The patient also experienced an episodic excruciating lancinating pain of the breast that would randomly and spontaneously radiate through either breast several times per day for 15 to 30 seconds. A workup including an antinuclear antibody test, complete blood cell count with differential, and comprehensive metabolic panel all were within reference range.

The patient was diagnosed with nipple vasospasm. Partial relief of nipple pain occurred after treatment with $30 \mathrm{mg}$ daily of nifedipine; $60 \mathrm{mg}$ daily resulted in complete control, allowing the patient to breastfeed without discomfort, but the lancinating pain continued unabated. The patient could not discontinue breastfeeding because her child was intolerant to formula. She became despondent, as she could find no relief from the pain that she found to be intolerable. Because the patient's description was reminiscent of the lancinating pain seen in postherpetic neuralgia, a trial of pregabalin was prescribed. A dosage of $75 \mathrm{mg}$ twice daily resulted in near-complete resolution of the pain. After 3 months, the patient successfully weaned her child from breast milk to formula, and the nipple and breast pain promptly resolved. The baby experienced no adverse effects from the patient's use of pregabalin.

This condition was first described by Gunther ${ }^{1}$ in 1970 as initial blanching of the nipple followed by a mulberry color. It was termed psychosomatic sore nipples. ${ }^{1}$

Dr. Gallego is from Sharp Rees-Stealy Medical Group, San Diego, California. Dr. Aleshaki is from St. Joseph Mercy Hospital, Ann Arbor, Michigan. The authors report no conflict of interest.

Correspondence: Humberto Gallego, MD (humbertogallego@yahoo.com). 
Lawlor-Smith and Lawlor-Smith ${ }^{2}$ described the condition in 1997 and termed it vasospasm of the nipple. They reported 5 patients who experienced debilitating nipple pain as well as the triphasic color change of Raynaud phenomenon or a biphasic color change (white and blue). Two patients had a history of Raynaud phenomenon affecting the digits before their first pregnancy. ${ }^{2}$ Anderson et $\mathrm{al}^{3}$ presented 12 breastfeeding women with Raynaud phenomenon of the nipple; only 1 patient had a history of Raynaud phenomenon. In this series, all 6 women who chose to try nifedipine responded well to the drug. ${ }^{3}$

Raynaud phenomenon of the nipple also has been reported to be associated with the use of labetalol. ${ }^{4}$ In this case, the patient had a history of Raynaud phenomenon affecting the toes and nipples on cold days. In 2 subsequent pregnancies she was treated with labetalol for pregnancyinduced hypertension, which resulted in severe nipple pain with each pregnancy unrelated to cold weather. Unlike other cases, this patient experienced antenatal symptoms in addition to the typical postnatal symptoms. The nipple pain resolved with discontinuation of the labetalol. ${ }^{4}$

Barrett et $\mathrm{al}^{5}$ conducted a retrospective review of medical records of 88 breastfeeding mothers who presented with nipple pain and dermatitis. They defined the criteria for Raynaud phenomenon of the nipple as chronic deep breast pain (in general lasting $>4$ weeks) that responded to therapy for the condition and had at least 2 of the following characteristics: (1) observed or self-reported color changes of the nipple, especially with cold exposure (white, blue, or red); (2) cold sensitivity or color changes of the hands or feet with cold exposure; or (3) failed therapy with oral antifungals. Using these criteria, they diagnosed 22 women $(25 \%)$ with Raynaud phenomenon of the nipple; 20 (91\%) reported a history of cold sensitivity or color change of acral surfaces. Of 12 patients who received and tolerated nifedipine use, $10(83 \%)$ reported decreased pain or complete resolution. This series described breast or nipple pain, whereas other reported cases only described nipple pain. The authors described a sharp, shooting, or stabbing painqualifications not previously noted. ${ }^{5}$ Our patient experienced both nipple pain and a lancinating breast pain consistent with the cases reported by Barrett et al. ${ }^{5}$

The nipple pain and treatment response in our patient was typical of previously reported cases of vasospasm of the nipple in breastfeeding women; however, Barrett et $\mathrm{a}^{5}$ did not describe individual patients who exhibited the dual nature of the pain described in our patient. The nipple pain experienced during breastfeeding in our patient was successfully treated with nifedipine. We report the successful treatment of the separate lancinating pain with pregabalin.

\section{REFERENCES}

1. Gunther M. Infant Feeding. London, United Kingdom: Methuen; 1970.

2. Lawlor-Smith L, Lawlor-Smith C. Vasospasm of the nipple-a manifestation of Raynaud's phenomenon: case reports. BMJ. 1997;314:644-645.

3. Anderson JE, Held N, Wright K. Raynaud phenomenon of the nipple: a treatable cause of painful breastfeeding. Pediatrics. 2004;113:360-364.

4. McGuinness N, CordingV. Raynaud's phenomenon of the nipple associated with labetalol use. J Hum Lact. 2013;29:17-19.

5. Barrett ME, Heller MM, Stone HF, et al. Raynaud phenomenon of the nipple in breastfeeding mothers: an underdiagnosed cause of nipple pain. JAMA Dermatol. 2013;149:300-306.

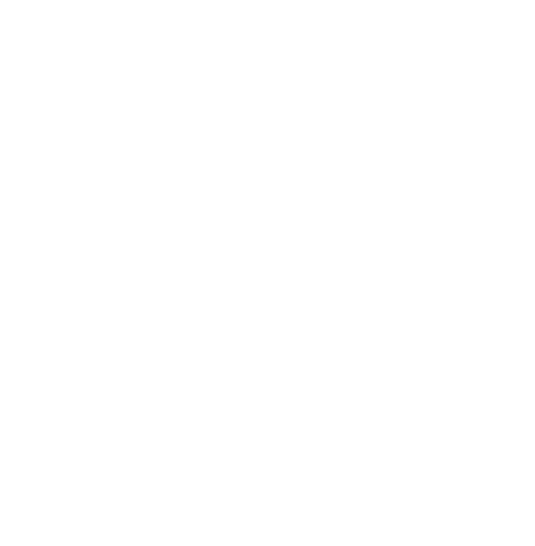

